\title{
Réanimation does not forget the foundations
}

\section{Réanimation does not forget the foundations}

\author{
A. Mercat \\ C SRLF et Springer-Verlag France 2014
}

L'analyse physiopathologique des situations de détresse vitale et des effets des interventions thérapeutiques a été et reste encore l'objet d'une recherche clinique intense et souvent passionnante. Cette approche, appliquée au quotidien dans le soin, a, pour beaucoup d'entre nous, joué un rôle déterminant dans la décision d'exercer le métier de réanimateur.

Dans une remise en cause argumentée de l'hégémonie de l'evidence-based medicine, trois éditorialistes de renom plaidaient pour que l'on n'oublie pas les fondements de la pratique médicale en général et de la réanimation en particulier que constituent la bonne connaissance de la physiologie et la compréhension de la physiopathologie [1]. Pour illustrer leurs propos, les auteurs rappelaient que David Ashbaugh et Tom Petty ont inventé le syndrome de détresse respiratoire aiguë (SDRA) en proposant une physiopathologie commune à la détresse respiratoire présentée par 12 patients atteints d'affections très diverses [2], ou encore que Paul Pepe et John Marini ont inventé la pression positive de fin d'expiration (PEEP) intrinsèque et décrit la physiopathologie de l'hyperinflation dynamique à partir de trois observations cliniques [3]. Ces deux exemples illustrent à mon sens parfaitement que, contrairement à ce que certains voudraient faire croire, le calcul de l'effectif nécessaire et la randomisation ne sont pas des préalables indispensables à toute recherche clinique pertinente et utile.
La décision du comité éditorial de consacrer un numéro entier de Réanimation à des articles de physiologie et/ou de physiopathologie me semble être parfaitement conforme à cette vision. Rompant avec la logique habituelle de regroupement thématique, ce numéro de la revue est constitué d'articles abordant des sujets très divers. Ces articles ont en commun d'apporter une synthèse actualisée des connaissances sur des mécanismes physiologiques ou physiopathologiques dont la compréhension est utile au médecin réanimateur, soit parce qu'ils expliquent les symptômes observés, soit parce qu'ils justifient l'approche thérapeutique. Le lecteur ne retrouvera dans ce numéro, j'en ai peur, ni forest plot ni cotation du « niveau de preuve ». Je suis convaincu qu'il saura s'en passer, préférant s'imprégner des concepts et analyses proposés.

\section{Références}

1. Brochard L, Mancebo J, Tobin M (2003) Searching for evidence: don't forget the foundations. Intensive Care Med 29:2109-11

2. Ashbaugh DG, Bigelow DB, Petty TL, Levine BE (1967) Acute respiratory distress in adults. Lancet 2:319-23

3. Pepe PE, Marini JJ (1982) Occult positive end-expiratory pressure in mechanically ventilated patients with airflow obstruction: the auto-PEEP effect. Am Rev Resp Dis 216:166-9
A. Mercat $(\square)$

Département de réanimation médicale et médecine hyperbare, CHU d'Angers, 4, rue Larrey, F-49933 Angers cedex 09

e-mail : almercat@chu-angers.fr 